\title{
Renal Cell Carcinoma and Prognostic Factors Predictive of Survival
}

\author{
M. Sorbellini, MD and G. Bratslavsky, MD \\ Urologic Oncology Branch, National Cancer Institute, National Institutes of Health, Bethesda, MD
}

We congratulate Haferkamp et al. ${ }^{1}$ for their work trying to identify prognostic factors predictive of survival for patients with renal cell carcinoma (RCC) who undergo nephron-sparing surgery for imperative indications. Although in the past decade, there has been a trend to move from radical nephrectomy to nephron preservation, many renal units are still lost unnecessarily. Identifying those patients at a higher risk for metastatic disease and those who are likely to fail local therapy is of paramount importance.

In the present study, the authors excluded patients with von Hippel-Lindau (VHL) syndrome, because these patients are prone to develop bilateral multifocal recurrent clear cell renal carcinomas. However, the reader should be aware that there are other hereditary syndromes besides VHL that predispose to the development of bilateral and/or multifocal renal cell carcinoma. These include patients with hereditary papillary renal carcinoma (HPRC) who develop bilateral papillary type $1 \mathrm{RCC}$, hereditary leiomyomatosis RCC (HLRCC) patients who tend to form aggressive papillary type 2 RCC, Birt-Hogg-Dube' (BHD) patients who may develop a whole spectrum of tumors from benign oncocytomas to chromophobe RCC to aggressive clear cell RCC, and several other less-recognized syndromes, such as tuberous sclerosis and succinatedehydrogenase-B deficiency. ${ }^{2}$ The above-mentioned syndromes may have multifocal RCC as a sole manifestation or as a constituent of a complex multiorgan involvement. Therefore, caution should be used in evaluating a study that excludes only VHL patients but not other hereditary forms of RCC. Additionally, there is a great variability of aggressiveness of lesions in patients with multifocal RCC.

(C) Society of Surgical Oncology 2009

Published Online: 12 November 2009

G. Bratslavsky, MD

e-mail: bratslag@mail.nih.gov
Unfortunately, the authors fail to delineate the type of patients who fit in the category of "imperative indications." Were there patients with solitary renal units due to contralateral nephrectomy or congenital absence of the contralateral kidney? If the contralateral kidney was lost due to cancer, which pathological variables were analyzed? Predicting cancer-specific survival from resection of a T1 renal mass from a solitary kidney may not be driving the oncologic outcome if the same patient had a contralateral nephrectomy for a T3 disease. Because 50\% of the cohort had bilateral disease, pathological information about contralateral kidney would be of great value and could provide additional predictors of poor outcome. Perhaps, a surprising finding of similar cancer-specific survival between T1, $\mathrm{T} 2$, and $\mathrm{T} 3$ groups may be explained by the confounding effects of contralateral pathology.

The authors also evaluated the effect of multifocality in their analysis. It has been shown that multifocal RCC is much more prevalent than originally believed, and ranges from $5-25 \%$ in the literature. ${ }^{3,4}$ The problem with the study of multifocal RCC is our current inability to determine whether a second renal tumor in the same or the contralateral kidney is indeed part of multifocal disease or simply a recurrence or metastasis from a previously operated tumor. Without a specific genetic study designed to evaluate the clonal origin of each mass found, it is impossible to determine whether patients have multifocal, recurrent, or metastatic disease. ${ }^{5-7}$ Had recurrences been counted as multifocality, the assignment of patients to the different groups in the study may have affected the statistical analysis. The opposite also could be true. Because approximately $20 \%$ of lesions are missed by current imaging techniques both preoperatively and intraoperatively, patients assigned to the unifocal group may have actually had multifocal disease. This would have influenced the assignment of patients into various groups being analyzed and ultimately accounted for the lack of statistical 
significance between unifocal vs. multifocal groups and unilateral vs. bilateral groups.

Although the present study evaluates patients spanning almost three decades, one of the major drawbacks is the fact that it may not have enough events to achieve statistical power. ${ }^{1}$ This is evident by the fact that well-known prognostic factors for cancer-specific survival (CSS), such as clinical stage (Tables 2, 3,4), did not achieve statistical significance in this study. Therefore, it is likely that a type II error is present in evaluating the variables responsible for CSS. Furthermore, the authors attempted to perform multivariate analyses of variables that were not significant on univariate analysis and the statistical validity of this analysis is questionable.

Finally, the authors found a "tendency" toward unfavorable prognosis in the case of positive surgical margins. Had there been more events, this tendency may have become statistically significant or completely disappeared. Moreover, the authors suggest doing everything in one's hands to avoid having positive surgical margins, including radical nephrectomy. Although we also strongly advocate appropriate surgery with negative surgical margins, the reader should be aware of recent data showing that positive surgical margins do not jeopardize survival outcomes in patients who undergo nephron-sparing surgery. ${ }^{8}$ In addition, loss of nephrons and its consequent renal insufficiency subjects patients to an increased risk for cardiovascular disease and death. ${ }^{9,10}$ Thus, we encourage surgeons to attempt nephron-sparing techniques whenever feasible.
ACKNOWLEDGEMENT This research was supported by the Intramural Research Program of the NIH, National Cancer Institute, Center for Cancer Research.

\section{REFERENCES}

1. Haferkamp A, Kurosch M, Pritsch M, et al. Prognostic factors influencing long-term survival of patients undergoing nephronsparing surgery for nonmetastatic renal-cell carcinoma (RCC) with imperative indications. Ann Surg Oncol. 2010. doi:10.1245/ s10434-009-0812-y

2. Sudarshan S, Linehan WM. Genetic basis of cancer of the kidney. Semin Oncol. 2006;33(5):544-51

3. Wunderlich H, Schlichter A, Zermann D, et al. Multifocality in renal cell carcinoma: a bilateral event? Urol Int 1999;63(3):160-3.

4. Kinouchi T, Mano M, Saiki S, et al. Incidence rate of satellite tumors in renal cell carcinoma. Cancer. 1999;86(11):2331-6.

5. Miyake H, Nakamura H, Hara I, et al. Multifocal renal cell carcinoma: evidence for a common clonal origin. Clin Cancer Res. 1998;4(10):2491-4.

6. Junker K, Thrum K, Schlichter A, et al. Clonal origin of multifocal renal cell carcinoma as determined by microsatellite analysis. J Urol. 2002;168(6):2632-6.

7. Blute ML, Itano NB, Cheville JC, et al. The effect of bilaterality, pathological features and surgical outcome in nonhereditary renal cell carcinoma. J Urol. 2003;169(4):1276-81.

8. Yossepowitch O, Thompson RH, Leibovich BC, et al. Positive surgical margins at partial nephrectomy: predictors and oncological outcomes. J Urol. 2008;179(6):2158-63.

9. Huang WC, Elkin EB, Levey AS, et al. Partial nephrectomy versus radical nephrectomy in patients with small renal tumors: is there a difference in mortality and cardiovascular outcomes? J Urol. 2009;181(1):55-62.

10. Sorbellini M, Kattan MW, Snyder ME, et al. Prognostic nomogram for renal insufficiency after radical or partial nephrectomy. J Urol. 2006;176(2):472-6. 